\title{
RASSF1A, the new guardian of mitosis
}

\author{
Endre Máthé
}

\begin{abstract}
The tumor suppressor gene RASSF1 contributes to the spatiotemporal regulation of mitosis through a new mechanism. By interacting with Cdc20, its protein product RASSF1A inhibits the anaphase-promoting complex and prevents degradation of cyclin $A$ and cyclin $B$ until the spindle checkpoint becomes fully operational.
\end{abstract}

Transcriptional silencing of the gene RASSF1 (Ras association domain family 1) has been observed in many cancers, and its tumor-suppression function has been implicated in reducing the proliferation of cancer cells ${ }^{1,2}$. RASSF1A has a Ras association domain like that of Ras effectors; it heterodimerizes with the Ras-GTP binding protein Nore1; and it is predicted to exert its function through a Ras signal transduction pathway ${ }^{3}$. Several interphase-specific functions have been proposed for RASSF1A, although the signaling pathways involved have not yet been identified ${ }^{3,4}$. In this month's issue of Nature Cell Biology, Min Sup Song and colleagues ${ }^{5}$ identified RASSF1A as a mitosis-specific inhibitor of the APC/C (anaphase-promoting complex/cyclosome), a large multisubunit complex that collaborates with ubiquitin-conjugating and ubiquitin-activating enzymes to catalyze the formation of polyubiquitin chains on its protein substrates, targeting them for degradation by the proteosome.

A number of regulatory mechanisms cooperate to restrict the activity of APC/C to a specific period in the cell cycle (Fig. 1). From $S$ phase to early prophase, the inhibitor Emi1 (early mitotic arrest 1 , also called regulator of cyclin A1 (Rcal)) predominates. APC/C binds first with Cdc20 (also called fizzy) and the complex APC/C-Cdc20 triggers protein degradation from prometaphase until telophase. From telophase to late G1, APC/CCdh1 is the active complex. In addition to the global activators Cdc20 and Cdh1 (Fzr), which are thought to act as substrate adaptors, local regulatory mechanisms are used by cells to promote or restrain the activity of APC/C to specific subcellular compartments.

The power of spindle checkpoint

One such mechanism, the spindle checkpoint, preserves genome integrity by keeping cells in metaphase until they have the correct chro-

Endre Máthé is at Cancer Research UK Cell Cycle Genetics Group, Department of Genetics, University of Cambridge, Downing Street, Cambridge, CB2 3EH, UK.

e-mail:em247@mole.bio.cam.ac.uk mosome alignment. In the case of unattached chromosomes or uneven tension across the sister chromatids, the kinetochores act as sensors and foster assembly of inhibitory proteins Mad2 and Mad3 onto APC/C or Cdc20, thereby halting the substrate-targeting and ubiquitinating activity of APC/C-Cdc20. When all chromosomes have their sister kinetochores attached to microtubules and face opposite spindle poles, the spindle checkpoint inhibition ceases, permitting APC/C-Cdc20 to initiate anaphase and sister-chromatid separation by ubiquitinating the separase inhibitor, securin. Additional regulatory mechanisms control local populations of APC/C-Cdc20, as it does not ubiquitinate all its substrates in a single sweep across the spindle and cytosol. Securin ubiquitination occurs when the chromosomes are at spindle equator, but cyclin B ubiquitination starts from the spindle poles.

\section{RASSF1A-Cdc20 connection}

The mitotic function of RASSF1A came to the attention of Song and colleagues ${ }^{5}$ when they tried to ectopically overexpress the gene in human cell lines and observed that these cells accumulated in prometaphase with levels of cyclin A and cyclin B remaining high. This suggested that the increased levels of RASSF1A arrested mitosis before the metaphase-anaphase transition. Indeed, depletion of RASSF1A by RNA interference (mimicking the naturally occurring gene silencing observed in tumors) accelerated mitotic progression. The destruction of cyclin A and cyclin B was initiated earlier in RASSF1A-deficient cells than in control cells, and these cells exited mitosis with severe chromosome segregation defects, suggesting that their spindle checkpoint was not operational. How can RASSF1A affect

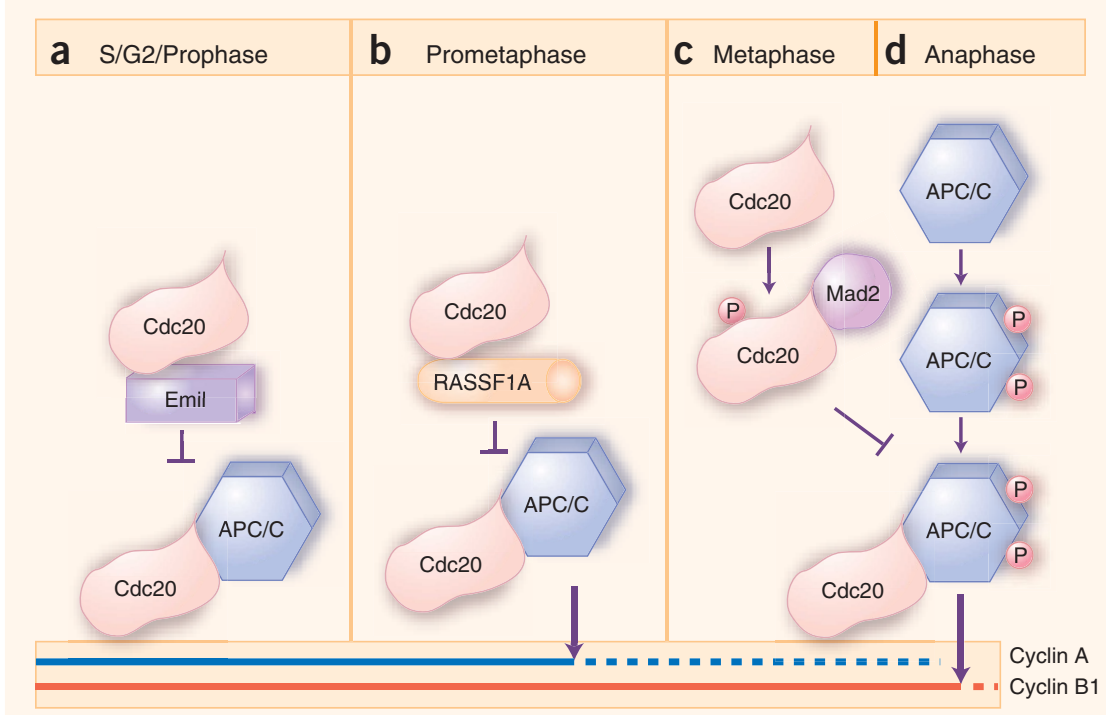

Figure 1 Inhibition of APC/C activity during the cell cycle. (a) From S phase to prophase, Emi1 inhibits the APC/C by binding to Cdc20. (b) During prometaphase, RASSF1A binds to Cdc20 and inhibits APC/C. At the end of prometaphase, this inhibition ceases and cyclin A degradation initiates. (c) During late prometaphase, the spindle checkpoint becomes activated. At kinetochores, phosphorylation and Mad2 binding will prevent $\mathrm{Cdc} 20$ from binding APC/C, with the exception of a certain population that supports cyclin A degradation. (d) Cyclin B remains stable as long as the spindle checkpoint is active. APC/C will become multiphosphorylated. The chromosomes become aligned and the spindle checkpoint is inactivated. As a consequence, nonphosphorylated Cdc20 binds to APC/C and anaphase inhibitors, including securin and cyclin $B$, are degraded. Blue and red solid lines indicate the stable stages for cyclin A and cyclin B1, respectively; dotted lines denote their degradation. 
mitotic progression? One clue to explain these abnormalities comes from Liu et al. ${ }^{6}$, who found that the C-terminal region of RASSF1A containing the Ras association domain bound to microtubules, conferring a strong cytoprotective activity against the microtubule-destabilizing drug nocodazol. At present, it is not clear whether altered microtubule stability is the primary cause of abnormal mitosis or the consequence of an unknown primary defect.

\section{A bridge between Emi1 and spindle checkpoint?}

Both Emil and the spindle checkpoint protein $\mathrm{Mad} 2$ negatively regulate APC/C through association with Cdc20, and the phenotypes resulting from RASSF1A overexpression or loss resemble those of cells in which Emil and Mad2 were similarly perturbed ${ }^{5}$. RASSF1A probably works in the same way, as RASSF1A and Cdc20 associate through their N-terminal regions and polyubiquitination activity of $\mathrm{APC} / \mathrm{C}-\mathrm{Cdc} 20$ is inhibited in vitro in the presence of full-length RASSF1A protein. Successive overexpression of RASSF1A and $\mathrm{Cdc} 20$ in vivo showed that the relative level of these antagonists is key in regulating APC/CCdc20 activity and the consequent destruction of both mitotic cyclins. A substantial amount of RASSF1A colocalized with Cdc20 at the poles and microtubules of prometaphase spindles, and coimmunoprecipitation experiments detected relatively low levels of association between RASSF1A and Cdc20 during mitosis. Thus, the new RASSF1A-Cdc20 regulatory mechanism operates during prometaphase.

Cells depleted of Emil do not accumulate mitotic cyclins or enter mitosis because APC/C activity is not properly inhibited. When RASSF1A was overexpressed in HeLa cells depleted of Emil, the mitotic cyclins accumulated and cells progressed into mitosis, arresting around prometaphase. Comparing the prophase peak of Cdc20Emil association to the prometaphase peak of Cdc20-RASSF1A association by immunoprecipitation from normal HeLa cells, the authors propose that RASSF1A is required for normal prometaphase progression, but its function is distinct and independent from that of Emil. This cannot fully explain how RASSF1A overexpression overcomes the effects of Emil depletion if their periods of Cdc20 association are different. It is possible that RASSF1A is also involved in Cdc20 regulation during the period in which Emil functions, but that its association with Cdc20 is more transient than it is in prometaphase, and so not detected by immmunoprecipitation from prophase extracts. If this is the case, more experiments are needed to investigate whether RASSF1A can regulate Cdc20 during prophase and the G2 phase of the cell cycle.

How is the prometaphase function for RASSF1A related to the spindle checkpoint? Lung cancer and HeLa cells, treated with nocodazol to activate their spindle checkpoint, stayed arrested in a prometaphase-like state with high levels of mitotic cyclins. The loss of RASSF1A protein had no apparent effect on this arrest, and levels of cyclin B remained unaffected. Because the cells did not exit mitosis in the presence of an active spindle checkpoint, the authors concluded that the RASSF1A function was not required for the normal checkpoint functioning. But when RASSF1A was overexpressed with the spindle checkpoint off, the cells did not exit mitosis and were still arrested in a prometaphase-like state, suggesting that excess RASSF1A could provide arrest in the absence of a spindle checkpoint signal.

The authors postulate that the spindle checkpoint and RASSF1A exert their negative control on APC/C-Cdc20 through independent mechanisms and at different time points. Indeed, the peak of Cdc20-RASSF1A and Cdc20-spindle checkpoint activities are different, indicating that they might operate at different time points or in different cellular compartments. Notably, cyclin A and cyclin B are not degraded in prometaphase-arrested cells generated by elevated levels of RASSF1A protein.

The activated spindle checkpoint prevents only the degradation of cyclin B and not that of cyclin A, whose APC/C-Cdc20-mediated destruction initiates after nuclear envelope breakdown in prometaphase. Thus it seems reasonable to speculate that the RASSF1Aassociated $\mathrm{Cdc} 20$ at the spindle apparatus is responsible for the inhibition of cyclin A degradation during prometaphase.

\section{The burden of Cdc20}

The spindle checkpoint is coordinated by the many partners of Cdc20, which binds kinetochores by its N-terminal region and centrosomes, spindle microtubules and $\mathrm{Mad} 2$ with its C-terminal region. It also uses the $\mathrm{N}$-terminal region to bind the TPR-subunits of APC/C and is degraded through its cyclin B-type destruction boxes and APC/C-Cdh1.

It is exciting to imagine a RASSF1A-dependent mechanism working like a cell cycle checkpoint. Some of the interactions required for this have now been demonstrated (for example, the binding of the $\mathrm{N}$-terminal region of $\mathrm{Cdc} 20$ to the $\mathrm{N}$-terminal regions of Emil and RASSF1A). The localization of RASSF1A to spindle poles, spindle microtubules and the cytosol could mean that this new molecular mechanism coordinates the activity of population(s) of Cdc20 and APC/C with that of the cytoskeleton or other cellular compartments.

Investigation of cells with both an activated spindle checkpoint and overexpressed RASSF1A, or abrogated checkpoint and diminished RASSF1A, might uncover the earliest steps of spindle checkpoint activation. Studies based on antibody injections in cultured cells can explore the mitotic functions of RASSF1A and Cdc20, advancing our understanding of how various substrates located at different compartments could be degraded simultaneously.

1. Dammann, R. et al. Nat. Genet. 25, 315-319 (2000).

2. Burbee, D.G. et al. J. Natl. Cancer Inst. 93, 691-699 (2001).

3. Ortiz-Vega, S. et al. Oncogene 21, 1381-1390 (2002).

4. Shivakumar, L. et al. Mol. Cell Biol. 22, 4309-4318 (2002)

5. Song, M.S. et al. Nat. Cell Biol. advance online publication, 25 January 2004 (doi: 10.1038/ncb1091).

6. Liu, L. et al. Oncogene 22, 8125-8136 (2003). 